\title{
Broadband, picosecond two-stage optical parametrical chirped pulse amplification system at $100 \mathrm{~Hz}$
}

\author{
Mario Galletti* \\ GoLP/Instituto de Plasmas e Fusão Nuclear, Instituto Superior Técnico, \\ Universidade de Lisboa, 1049-001 Lisboa, Portugal \\ Giedre Archipovaite, Pedro Oliveira, Marco Galimberti, \\ Ian Musgrave, and Cristina Hernandez-Gomez \\ Central Laser Facility, Science and Technology Facilities Council, Rutherford Appleton Laboratory, \\ Harwell Science and Innovation Campus, Didcot OX11 OQX, United Kingdom
}

(Received 18 December 2018; published 20 May 2019)

\begin{abstract}
We report on a broadband, picosecond two-stage optical parametrical chirped pulse amplification system, pumped at $515 \mathrm{~nm}$ by a frequency doubled $\mathrm{Yb}$ regenerative amplifier. The system, designed to be part of the new petawatt picosecond front end, delivers $30 \mu \mathrm{J}$ pulses at a central wavelength of $870 \mathrm{~nm}$ with about $200 \mathrm{~nm}$ bandwidth at $100 \mathrm{~Hz}$. The overall efficiency of the system (pump signal) is $8 \%$ while the second stage presents $\sim 17 \%$ conversion efficiency.
\end{abstract}

DOI: 10.1103/PhysRevAccelBeams.22.051301

\section{INTRODUCTION}

Intense ultrashort pulses are an attractive resource for a wide range of experiments, contributing significantly to the advancement of new research areas like laboratory astrophysics [1], high energy density physics [2], schemes for particle acceleration [3,4], and so on. This kind of experimental campaigns requires laser drivers with peak power of 100's of TW to PW, less than 100 fs pulse duration regime [5-10] with tunable central wavelength because of maximizing the $a_{0} \propto I \lambda^{2}$ (normalized laser potential). Moreover, also a laser system with high repetition rate, in the $\mathrm{mJ}$ energy range and tunable, can meet the needs of the accelerator community, among others in laser-induced FEL seeding and FEL pump-probe experiments [11].

The chirped-pulse amplification (CPA) [12] method was initially developed for the ultrashort pulse amplification with laser amplifiers, but in the past few decades the method was generalized to be applied to optical parametric amplifiers (OPAs) [13], as OPCPA.

The OPCPA has a number of important advantages with respect to a classical CPA based on laser gain media

\footnotetext{
mario.galletti@stfc.ac.uk

Also at Central Laser Facility, Science and Technology Facilities Council, Rutherford Appleton Laboratory, Harwell Science and Innovation Campus, Didcot OX11 0QX, United Kingdom.

Published by the American Physical Society under the terms of the Creative Commons Attribution 4.0 International license. Further distribution of this work must maintain attribution to the author(s) and the published article's title, journal citation, and DOI.
}

[14-16]. The parametric gain in the nonlinear crystal, within a single pass, can be as high as $10^{10}$ [17]. The gain medium can also exhibit a bigger gain by unit of length, which can reduce the $\mathrm{B}$ integral and material dispersion; all of this leads to simpler and compact setups. However, OPCPA amplification only occurs within the duration of the pump pulse, this allows for a bigger contrast outside the pump pulse duration and allows for temporalspectral control of the amplified pulse.

Moreover, the amplification is possible in a wide range of wavelengths, ranging from the visible to the mid-IR, and fulfilling certain optimized phase-matching conditions, like in noncollinear geometries, the supported bandwidth can be very large allowing very short high-energy pulses to be generated.

Thermal effects in the parametric amplification process are much weaker than in a laser gain media because of the instantaneous nature of the process only a small amount of heating due to weak parasitic absorption is present. The absence of thermal effect combined with the very high quantum efficiency allows for scaling to very high energy and peak power levels, and also to a high beam quality of the amplified pulses.

Finally, the parametric amplification preserves the initial property of the signal pulse and sometimes the generated idler wave can also be used.

However, the OPCPA concept presents some disadvantages/setup difficulties with respect to the classical CPA with laser amplifiers such as: the temporal duration matching and synchronization between the pump and signal pulses to maintain high gain; the high quality of the pump beam; the limited aperture of most available nonlinear crystals in highenergy stages and the demanding phase-matching conditions. 
At the central laser facility (RAL), a new PW laser beam line for the Vulcan laser is being developed, matching the requirements needed from the laser-based accelerator community. This will be fully based on OPCPA as an amplification technique and it will demonstrate the technology feasibility for larger systems opening the path for a ultrashort pulse system limited exclusively by the pump laser available. This system will aim at providing a tunable multi-TW to PW laser system for pump-probe experiments with the already existing Vulcan PW laser, betatron imaging and laser-matter acceleration experiment. The laser system is going to deliver $30 \mathrm{~J}$ in $30 \mathrm{fs}$ (or less) on target, at a "noncommon" central wavelength of $870 \mathrm{~nm}$ for large scale laser system (off-harmonic respect to the PW Vulcan laser).

Such systems employ multiple amplification stages, with the first amplification stages (picosecond temporal regime, multi- $\mu \mathrm{J}$ to multi-mJ level) typically based on a bariumborate oxide crystal (BBO) [18], well studied and successful for near-IR amplification. Lithium Triborate (LBO) or Potassium dihydrogen phosphate (KDP) [18] are not studied so extensively, however they can be used up to the final amplification stages (nanosecond temporal regime, multi-J level) because of the possibility to fabricate these crystals with useful apertures on the order of $100 \mathrm{~mm}$ and few tens centimeters in length.

Because of this reason, we choose to implement LBO crystals in all the OPA stages through the system, in particular in the low energy ps pulse length stages. Finally, it will ensure successful performances of the last amplification stages and the homogeneity/robustness of the operational PW system.

We present a compact, broadband two-stage Noncollinear optical parametric amplifier (NOPA) system, with good temporal and output power stability, part of the fully
OPCPA new Vulcan beam line. To the best of our knowledge, it is the first operational system working at $870 \mathrm{~nm}$ adopting a front end based on LBO crystals to maintain the full amplified broad bandwidth and homogeneity/ robustness throughout all the system.

The low energy ps front end consists of two LBO OPA stages delivering pulses with $870 \mathrm{~nm}$ central wavelength, energies of $30 \mu \mathrm{m}$ at $100 \mathrm{~Hz}$ repetition rate.

The OPAs stages are seeded by a commercial titaniumsapphire oscillator and pumped at $515 \mathrm{~nm}$ by an optically synchronized frequency doubled $\mathrm{Yb}$ regenerative amplifier. The overall efficiency of the system (pump signal) is $8 \%$ while the second stage presents $\sim 17 \%$ conversion efficiency.

\section{EXPERIMENTAL SETUP}

The presented broadband, double-stage OPA system at $100 \mathrm{~Hz}$ repetition rate is based on a $\mathrm{Yb}$ regenerative amplifier design. This concept enables a very compact $(75 \mathrm{~cm} \times 210 \mathrm{~cm})$ OPCPA system with reduced complexity (see Fig. 1).

The OPA system is seeded by a $80 \mathrm{MHz}$ Ti:sapphire oscillator (Venteon, Laser Quantum), which delivers nJ-level, sub-6 fs pulses with an ultrabroadband spectrum from 700 to $1150 \mathrm{~nm}$.

By use of a dichroic mirror the output spectrum of the Ti:Sa oscillator is divided into two parts: a broadband spectrum spanning from 700 to $1000 \mathrm{~nm}$, supporting sub- 6 fs pulse duration, while the infrared part in the second arm provides sufficient power at $1030 \mathrm{~nm}$ for seeding a fiber-based preamplifier (Venteon pre-amp3). The output of this fiber preamplifier seeds a Yb regenerative amplifier (S-pulse, Amplitude Systems), shown as pump laser in Fig. 1.

The output energy of this pump is $1.7 \mathrm{~mJ}$ per pulse and the pulse duration is $12 \mathrm{ps}$ with a central wavelength of

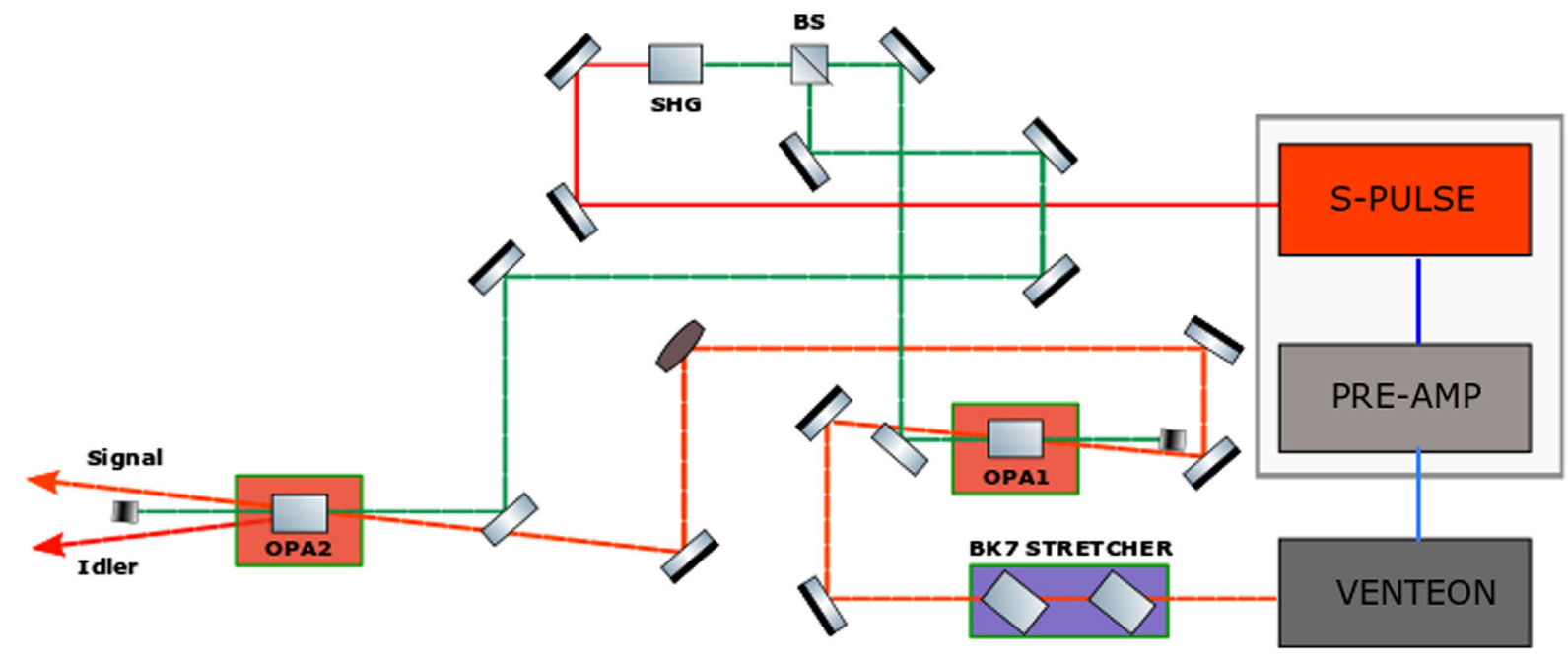

FIG. 1. The experimental setup sketch. Ti:Sa octave spanning (Venteon Oscillator), preamplifiers and regenerative amplifier (pump laser) respectively the seed and pump the double NOPAs setup. The violet box shows the seed compact stretcher made by double BK7 glass blocks. Highlighted: SHG—second harmonic generation stage; BS—beam splitter; OPA1/2—amplification stages. 

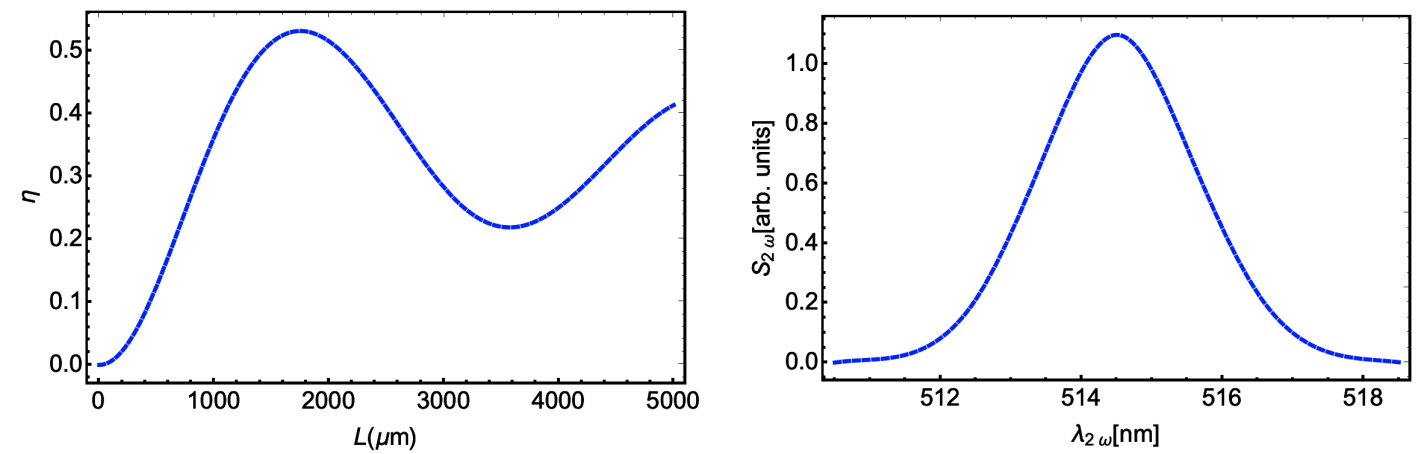

FIG. 2. Simulation on SHG nonlinear crystal properties: (right) BBO doubling efficiency vs crystal length and (left) SHG spectrum supported by BBO crystal.

$1030 \mathrm{~nm}$ and a repetition rate of $100 \mathrm{~Hz}$. The S-pulse amplifier output was frequency doubled with $\sim 50 \%$ efficiency in a $2 \mathrm{~mm}$ long BBO crystal. After the doubling stage the green beam pulse duration is about $10 \mathrm{ps}$ with a measured bandwidth of $1.7 \mathrm{~nm}$. The choice of the BBO as a doubling crystal was made after verifying the efficiency (Fig. 2, right) and supported bandwidth (Fig. 2, left) vs crystal length by an in-house developed numerical simulation code solving the three-wave mixing equation in the second harmonic generation (SHG) regime.

Subsequently, the frequency doubled beam is equally split in two for the NOPA stages pumping. The pump energies measured right before the amplification stages were 200 and $210 \mu \mathrm{J}$ for OPA1 and OPA2 respectively.

The OPA seed is the broadband beam delivered by the Ti: $\mathrm{Sa}$ Venteon oscillator stretched in a double pass stretcher. The stretcher consists of two $(50 \times 50 \times 50) \mathrm{mm}^{3}$ BK7 cubes both set at the Brewster angle. The estimated stretched signal pulse duration is $3 \mathrm{ps}$. In order to optimize the amplification efficiency and the signal quality, the seed pulse duration is maintained shorter than the pump, which is around $10 \mathrm{ps}$. The pump size is set smaller than the signal size to minimize the generation of superfluorescence.

The two single-pass OPAs stages are based on $(7 \times 7 \times$ 19) $\mathrm{mm}^{3}$ and $(7 \times 7 \times 20) \mathrm{mm}^{3}$ LBO crystals, both noncollinearly phase matched at $870 \mathrm{~nm}$ for broadband amplification of the fundamental Ti:sapphire oscillator seed spectrum.

The two-stage system is designed to be an operational system. For this reason, the intensity is fixed at $\mathrm{GW} / \mathrm{cm}^{2}$

TABLE I. Main parameters of the system.

\begin{tabular}{lccc}
\hline \hline & $\tau[\mathrm{ps}]$ & $\mathrm{E}$ & Crystal length [mm] \\
\hline VENTEON & $6 \times 10^{-3}$ & $\sim \mathrm{nJ}$ & $\ldots$ \\
S-PULSE & 12 & $\sim 1.7 \mathrm{~mJ}$ & $\ldots$ \\
SHG & 10 & $\sim 0.8 \mathrm{~mJ}$ & $2(\mathrm{BBO})$ \\
STRETCHER & 3 & $\sim \mathrm{nJ}$ & $200(\mathrm{BK} 7)$ \\
OPA1 & 3 & $\sim 100 \mathrm{~nJ}$ & 19 (LBO) \\
OPA2 & 3 & $\sim 30 \mu \mathrm{J}$ & 20 (LBO) \\
\hline \hline
\end{tabular}

level (equal splitting of the pump). Relaxing the condition related to the intensity and having OPA stages based on LBO $\left(d_{\text {eff }}=0.8 \mathrm{pm} / \mathrm{V} @ 870 \mathrm{~nm}\right)$, the crystal length should be increased. According to the OPA gain calculation (considering spatial walk-off), a $20 \mathrm{~mm}$ LBO crystal (adopting our geometry) presents broad-bandwidth amplification of $\sim 200 \mathrm{~nm}$. The main parameters of all the system stages are resumed in Table I.

\section{OPTICAL PARAMETRIC AMPLIFICATION RESULTS}

Broadband phase matching, 750-1000 nm spectral range, is ensured by choosing a determined noncollinear geometry between pump and signal beam, corresponding at $2.51^{\circ}$ external angle. This result was quantitatively confirmed by numerical simulations solving the three-wave mixing equation in the parametric amplification regime [19], as shown in Fig. 3.

A good temporal overlap between the seed and the $10 \mathrm{ps}$ pump pulses should be set to ensure good efficiency during the amplification process. As previously mentioned, a bulk stretcher was used in front of the first OPA stage resulting in a signal pulse duration of approximately $3 \mathrm{ps}$. Both parametric amplification stages are based on the same single-pass noncollinear geometry $\left[\theta_{\mathrm{PM}} \sim 14.3^{\circ}\right.$ and $\theta_{\text {sig }} \sim$ $2.51^{\circ}$ (external)] to ensure broadband amplification. In the first OPA stage, pumped with $200 \mu \mathrm{J}, 10$ ps long pulses, a high gain single-pass amplification from $\sim \mathrm{nJ}$ to $\sim 100 \mathrm{~nJ}$ is shown. The amplified bandwidth is $\sim 100 \mathrm{~nm}$, in Fig. 4 (green area). The amplified beam was injected in the second amplification stage by means of an achromatic beam transport system that maintains the broad bandwidth. For the second amplification stage, maintaining the same pumping system parameters, the output has an energy up to $30 \mu \mathrm{J}$ and a further broader spectrum, as shown in Fig. 4 (blue area).

The pump-signal temporal delay has been optimized for maximum output power and broadband spectrum for each stage fixed the geometry. The difference in the amplified spectrum by each stage is given by the different regime in 


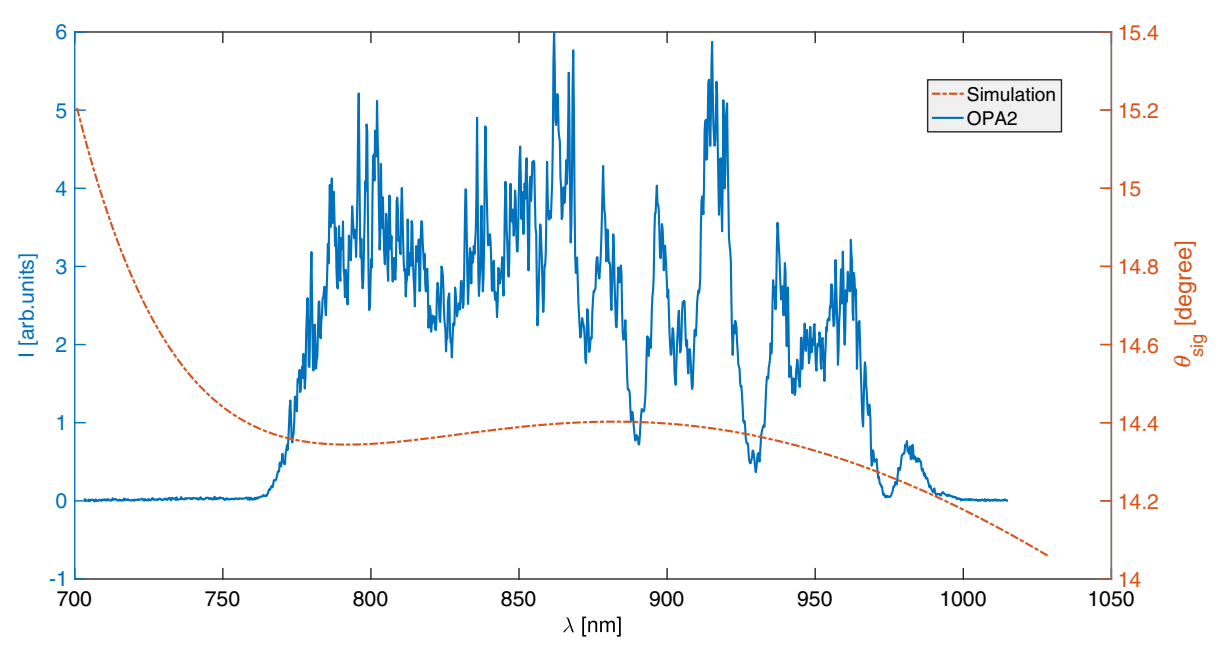

FIG. 3. Phase matching at 2.51 noncollinear external angle and crystal temperature at $40^{\circ} \mathrm{C}$ superimposed to experimental results regarding the OPA2.

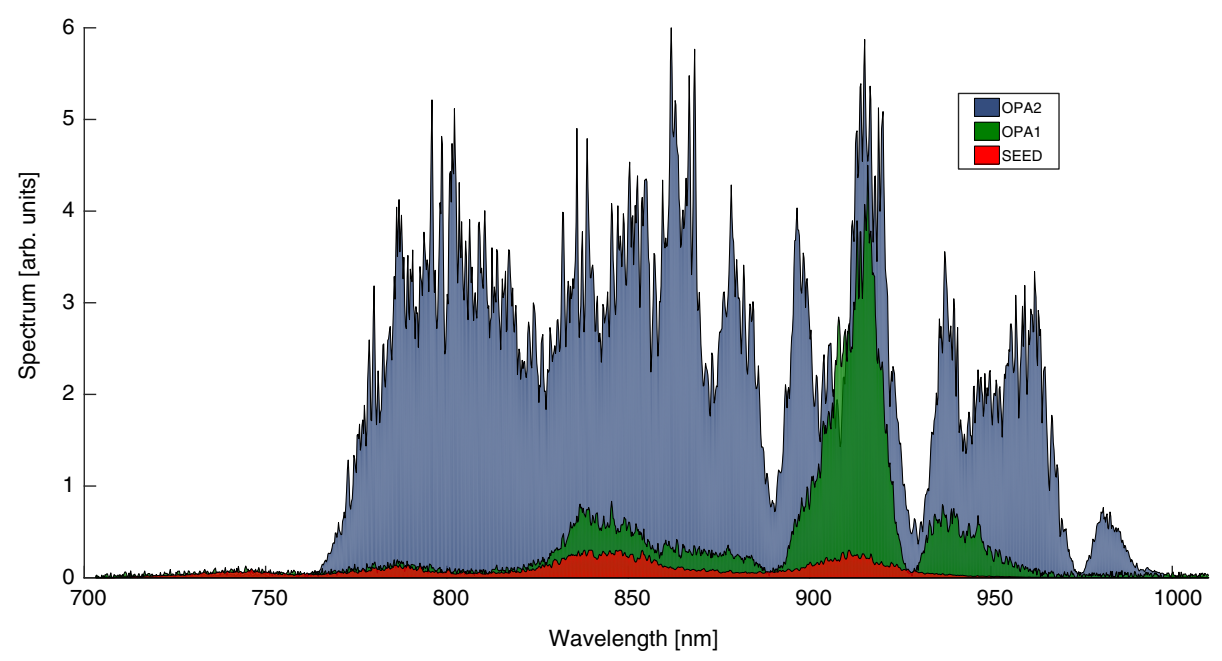

FIG. 4. Spectra related to the signal, amplified signal after the first OPA stage and after the second OPA stage.

which they are working; meanwhile the first one is not in the saturation regime, the second one is. The "pump to signal" efficiency of the second OPA is $17 \%$. The output spatial profile is Gaussian, shown in Fig. 5. Stability measurements were performed both on pulse spectrum [Fig. 6(a)] and output power stability [Fig. 6(b)]. They are stable for over 20 minutes and the rms values are 1\%, 7.3\% and $4.3 \%$ respectively for SHG, OPA1 and OPA2. The rms stability could be improved by implementing an active stabilization of the temporal delay for both amplification stages. On longer timescales power drifts were observed which could be corrected by minor adjustment of the delay stage relative to the pump of the OPAs stage crystals.

Regarding the spectrum stability, just the spectrum of NOPA1 was recorded because the first stage is not in the saturation regime (less stable), while the second stage is in the saturation regime (stable broadband spectrum). For the case that the signal in front of the first OPA stage is blocked, the single-pass stage generates insignificant superfluorescence background (Fig. 7). The amount of superfluorescence is much lower in the seeded than in the

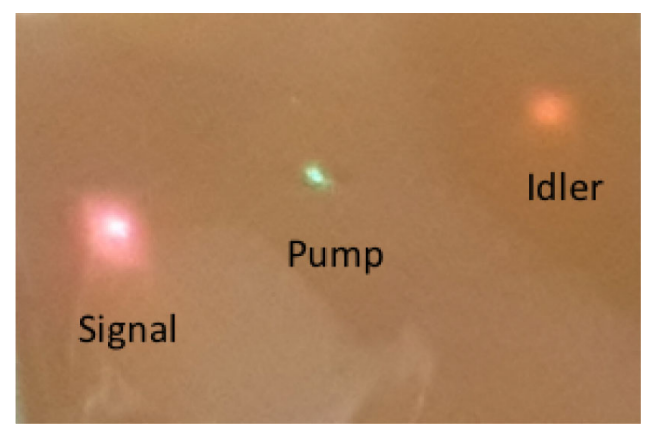

FIG. 5. OPA2 output beams: signal, idler and undepleted pump. 

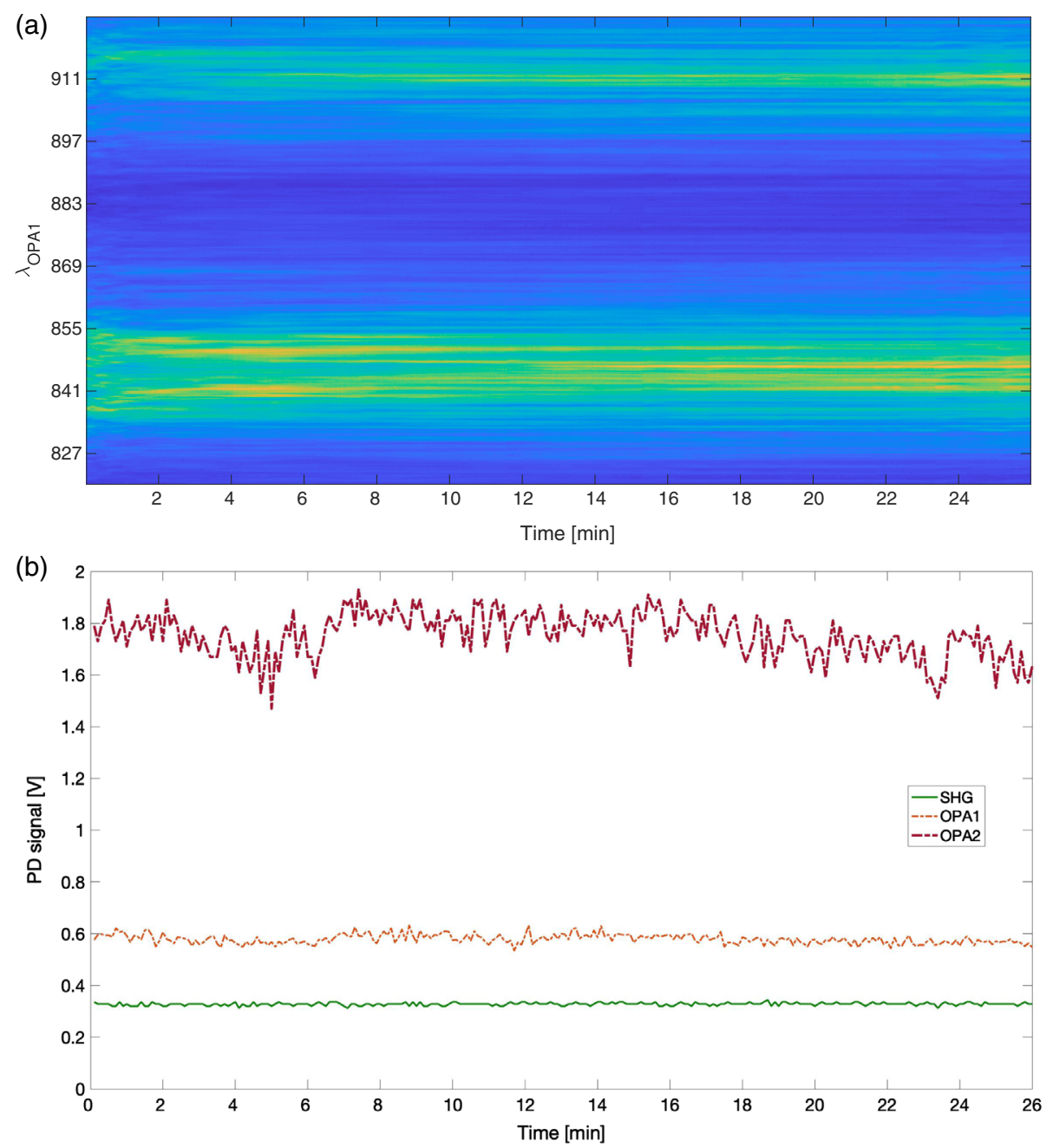

FIG. 6. Stability measurement performed for about 25 minutes: (a) OPA1 spectrum; (b) SHG, OPA1 and OPA2 output power. OPA1 PD signal is increased by a factor 0.3 to be better represented.

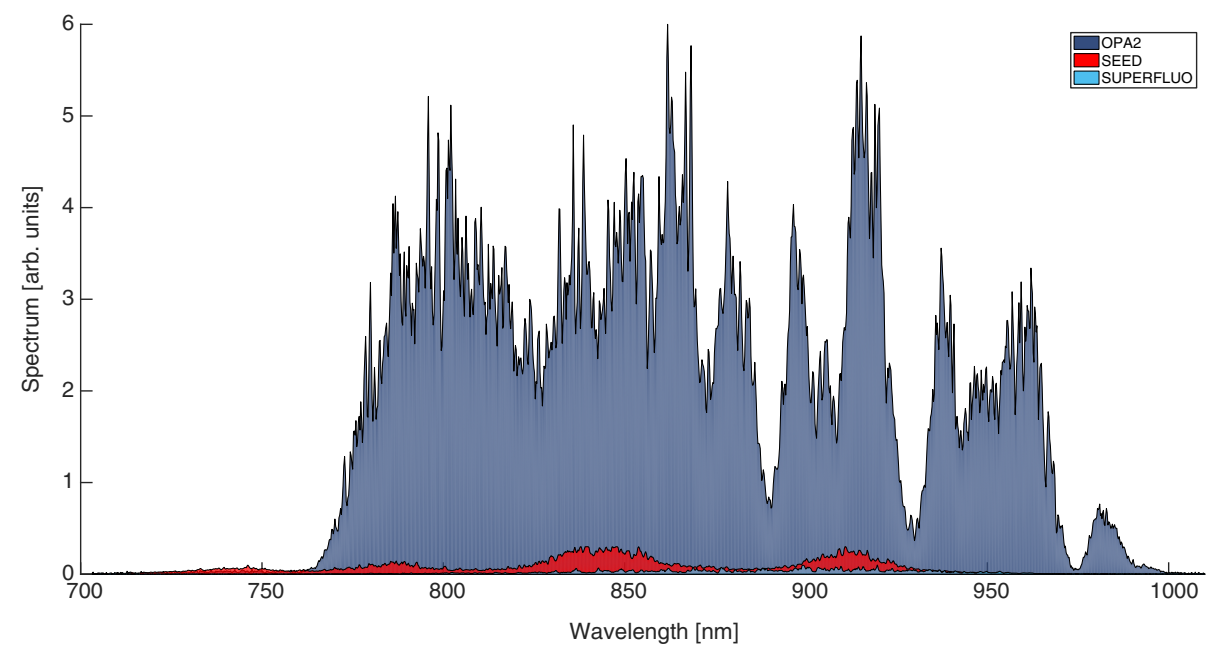

FIG. 7. Spectrum of the parametric superfluorescence compared to the oscillator and OPA2 spectrum. 
unseeded case. No further measurements were performed on the superfluorescence because of this reason.

\section{CONCLUSION}

The concept presented here is suited to realize a multi- $\mu \mathrm{J}$, sub-10 fs source with a high repetition rate (RR), depending on the RR of the pumping system employed, which comes with a significant reduction of setup complexity in comparison with other state-of-the-art OPCPA systems.

In our project devoted to develop a system with the pulse peak power reaching PW level and maintaining the sub$30 \mathrm{fs}$ pulse duration mainly for laser-based acceleration and pump-probe experiments, this compact setup is suited to perform as a $\mu \mathrm{J}$-level ps double stage OPA for the laser system front end, operating in a stable power and spectral regime.

It is based on an ultrabroadband Ti:sapphire seed oscillator with all-optical synchronization scheme to an efficient CPA solid-state pump source. The 10 ps amplifier output could be easily frequency doubled in a single-pass setup with high efficiency. Parametric amplification in two single-pass noncollinear amplifier stages leads to ultrabroadband amplification of $30 \mu \mathrm{J}$ of pulse. We have implemented the LBO crystal into the $\mu \mathrm{J}$ level OPCPA to confirm that it can successfully support broadband amplification at $870 \mathrm{~nm}$ and could be further implemented up to the final stages of the PW OPCPA system.

\section{ACKNOWLEDGMENTS}

M. Galletti acknowledges the financial support of the European Union's Horizon 2020 research and innovation program under Grant Agreement No. 654148 (LaserlabEurope), the Euratom research and training program under Grant Agreement No. 633053 and the Fundação para a Ciencia e a Tecnologia (FCT, Lisboa) under Grant No. PD/ BD/114327/2016. The work of M. Galletti was carried out in the framework of the Advanced Program in Plasma Science and Engineering (APPLAuSE, sponsored by FCT under Grant No. PD/00505/2012) at Instituto Superior Técnico (IST). Fundação para a Ciencia e a Tecnologia

[1] B. A. Remington, D. Arnett, R. Paul, H. Takabe et al., Modeling astrophysical phenomena in the laboratory with intense lasers, Science 284, 1488 (1999).

[2] M. Roth, T. Cowan, M. Key, S. Hatchett, C. Brown, W. Fountain, J. Johnson, D. Pennington, R. Snavely, S. Wilks et al., Fast Ignition by Intense Laser-Accelerated Proton Beams, Phys. Rev. Lett. 86, 436 (2001).

[3] T. Bartal, M. E. Foord, C. Bellei, M. H. Key, K. A. Flippo, S. A. Gaillard, D. T. Offermann, P. K. Patel, L. C. Jarrott, D. P. Higginson et al., Focusing of short-pulse high-intensity laser-accelerated proton beams, Nat. Phys. 8, 139 (2012).
[4] K. Ledingham and W. Galster, Laser-driven particle and photon beams and some applications, New J. Phys. 12, 045005 (2010).

[5] I. Ross, J. Collier, P. Matousek, C. Danson, D. Neely, R. Allott, D. Pepler, C. Hernandez-Gomez, and K. Osvay, Generation of terawatt pulses by use of optical parametric chirped pulse amplification, Appl. Opt. 39, $2422(2000)$

[6] X. Yang, Z.-Z. Xu, Y.-X. Leng, H.-H. Lu, L.-H. Lin, Z.-Q. Zhang, R.-X. Li, W.-Q. Zhang, D.-J. Yin, and B. Tang, Multiterawatt laser system based on optical parametric chirped pulse amplification, Opt. Lett. 27, 1135 (2002).

[7] C. N. Danson et al., Vulcan petawatt: Design, operation and interactions at $5 \times 10^{20} \mathrm{Wcm}^{-2}$, Laser Part. Beams 23, 87 (2005).

[8] Y. Kitagawa et al., Prepulse-free petawatt laser for a fast ignitor, IEEE J. Quantum Electron. 40, 281 (2004).

[9] V. Lozhkarev, G. Freidman, V. Ginzburg, E. Katin, E. Khazanov, A. Kirsanov, G. Luchinin, A. Malshakov, M. Martyanov, O. Palashov, A. Poteomkin, A. Sergeev, A. Shaykin, I. Yakovlev, S. Garanin, S. Sukharev, N. Rukavishnikov, A. Charukhchev, R. Gerke, and V. Yashin, 200 TW 45 fs laser based on optical parametric chirped pulse amplification, Opt. Express 14, 446 (2006).

[10] L. Waxer, D. Maywar, J. Kelly, T. Kessler, B. Kruschwitz, S. Loucks, R. McCrory, D. Meyerhofer, S. Morse, C. Stoeckl, and J. Zuegel, High-energy petawatt capability for the omega laser, Opt. Photonics News 16, 30 (2005).

[11] H. Hoppner, A. Hage, T. Tanikawa, M. Schulz, R. Riedel, U. Teubner, M. J. Prandolini, B. Faatz, and F. Tavella, An optical parametric chirped-pulse amplifier for seeding high repetition rate free-electron lasers, New J. Phys. 17, 053020 (2015).

[12] D. Strickland and G. Mourou, Compression of amplified chirped optical pulses, Opt. Commun. 56, 219 (1985).

[13] A. Dubietis, G. Jonusauskas, and A. Piskarskas, Powerful femtosecond pulse generation by chirped and stretched pulse parametric amplification in BBO crystal, Opt. Commun. 88, 437 (1992).

[14] A. Dubietis, R. Butkus, and A. P. Piskarskas, Trends in chirped pulse optical parametric amplification, IEEE J. Sel. Top. Quantum Electron. 12, 163 (2006).

[15] S. Witte and K. S. E. Eikema, Ultrafast optical parametric chirped-pulse amplification, IEEE J. Sel. Top. Quantum Electron. 18, 296 (2012).

[16] M. J. Prandolini, R. Riedel, M. F. Schulz, and F. Tavella, A review of high power OPCPA technology for high repetition rate freeelectron lasers, Proceedings of FEL2014, Basel, Switzerland, (JACoW, Geneva, Switzerland, 2014).

[17] I. Musgrave, W. Shaikh, M. Galimberti, A. Boyle, C. Hernandez-Gomez, K. Lancaster, and R. Heathcote, Picosecond optical parametric chirped pulse amplifier as a preamplifier to generate high-energy seed pulses for contrast enhancement, Appl. Opt. 49, 6558 (2010).

[18] D. N. Nikogosian, Nonlinear Optical Crystals: A Complete Survey (Springer, New York, NY, USA, 2005).

[19] A. Smith, http://www.as-photonics.com/snlo. 